\title{
BORON PARTICLES PRODUCTION IN NONEQUILIBRIUM LASER-CHEMICAL RADICAL REACTIONS DURING THE IR MULTIPHOTON DISSOCIATION OF $\mathrm{HClC}=\mathrm{CBCl}_{2} \mathrm{H}$ MOLECULES
}

\author{
G. I. ABDUSHELISHVILI, T. G. ABZIANIDZE, \\ A. G. BAKHTADZE, P. D. KERVALISHVILI, \\ G. I. TKESHELASHVILI and T. B. TZINADZE
}

Scientific Research Institute of Stable Isotopes, Kavtaradze Street, N21, Tbilisi 380 086, Georgia, USSR

(Received 20 February, 1988; in final form 6 May, 1988)

\begin{abstract}
The study of IR MPD of $\mathrm{HClC}=\mathrm{CBCl}_{2} \mathrm{H}$ molecule by $\mathrm{CO}_{2}$-laser is presented. We provide experimental results showing high degree of fragmentation of this molecule (down to elementary boron) during nonequilibrium laser induced radical bimolecular reactions.
\end{abstract}

KEY WORDS: Multiphoton dissociation, $\mathrm{CO}_{2}$-laser. $\mathrm{HClC}=\mathrm{CBCl}_{2} \mathrm{H}$ molecules, nonequilibrium laserchemical reactions, elementary boron.

\section{INTRODUCTION}

The method of multiphoton dissociation (MPD) of molecules in high-power pulsed IR-laser radiation field has lately found wide application in physico-chemical molecular processes. ${ }^{1-3}$ More specifically it is an effective procedure for producing high concentrations of free radicals. These radicals may be used for initiating nonequilibrium gas-phase radical reactions. A wide variety of final products is known to be produced during thermally initiated gas-phase radical reactions of multiatomic molecules, the desired product yield being quite low. This is due to the large number of possible reaction channels of the multicomponent mixtures in thermodynamic equilibrium, when temperatures of the initial, intermediate and final products are almost equal. The situation is different for nonequilibrium reactions where these temperatures may differ widely. High specificity of IR-laser radical reactions, i.e. high yield of the desired final product and suppression of other products, was mentioned in literature. ${ }^{4}$

In this paper we discuss the investigation of the MPD of chlorethylendichlorborane molecules $\left(\mathrm{HClC}=\mathrm{CBCl}_{2} \mathrm{H}\right)$ in radiation field of pulsed $\mathrm{CO}_{2}$-laser. Basic interest in this molecule is due to the prospects of its application as a material for laser induced isotope separation. ${ }^{5-7}$ In this paper we provide experimental results showing high fragmentation of $\mathrm{HClC}=\mathrm{CBCl}_{2} \mathrm{H}$ molecules (down to elementary boron) in the IR laser radiation field during nonequilibrium laser induced radical bimolecular reactions. Some of the data were published previously. ${ }^{8-10}$ 


\section{EXPERIMENTAL SECTION}

IR MPD of $\mathrm{HClC}=\mathrm{CBCl}_{2} \mathrm{H}$ molecules in proper gas in the field of pulsed $\mathrm{CO}_{2}$-laser was studied experimentally. Pulsed TEA $\mathrm{CO}_{2}$-laser with line selection was used. In all the experiments the laser was tuned to resonance frequency $v=986.6 \mathrm{~cm}^{-1}$ of $\mathrm{B}-\mathrm{Cl}$ bond of $\mathrm{HClC}=\mathrm{C}^{10} \mathrm{BCl}_{2} \mathrm{H}$ molecules. Laser pulse duration was varied by changing the actuating medium composition. Typical pulse specifications: pulse duration at the half-height of the head of pulse $\sim 100 \mathrm{~ns}$, pulse duration at the base- $-1 \mu \mathrm{s}$; pulse head to pulse tail power ratio-70:30; energy density $\phi \simeq 2.5$ $\mathrm{J} / \mathrm{cm}^{2}$. The experiments were carried out mainly in the flow-through mode. Stainlesssteel cell with monocrystal $\mathrm{NaCl}$ windows was used. The pumped gas molecules entered the reactor via the distributor and after forming a laminar flow entered the reaction zone crossing the laser beam pulse (Figure 1). Pumping rate was selected so that during the delay time between pulses the irradiated gas portions could be completely removed from the reaction zone (ideal displacement reactor ${ }^{11}$ ). Gas composition before and after irradiation was determined from the IR absorption spectra in the "SPECORD-75 IR" spectrophotometer.

The absorbed energy dependence on the laser radiation energy density, and dissociation yield reduction with buffer gas addition were studied in the static mode in the individual experiments.

\section{EXPERIMENTAL RESULTS AND DATA PROCESSING}

Figure 2 shows characteristic IR spectrogram of the original material and decay products. Absorption peaks of the $\mathrm{BCl}_{3}$ and $\mathrm{C}_{2} \mathrm{H}_{2}$ decay products are clearly observable. When large volumes of the material were processed, dichlorethylene $\mathrm{C}_{2} \mathrm{H}_{2} \mathrm{Cl}_{2}$ absorption peaks were also measured. Besides when pumping large

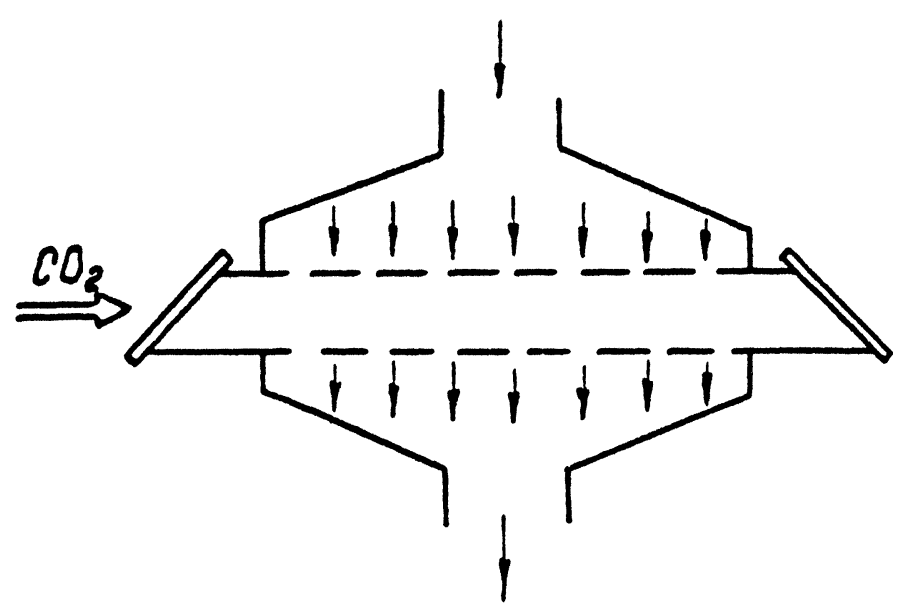

Figure 1 Laser reactor schematic diagram. 


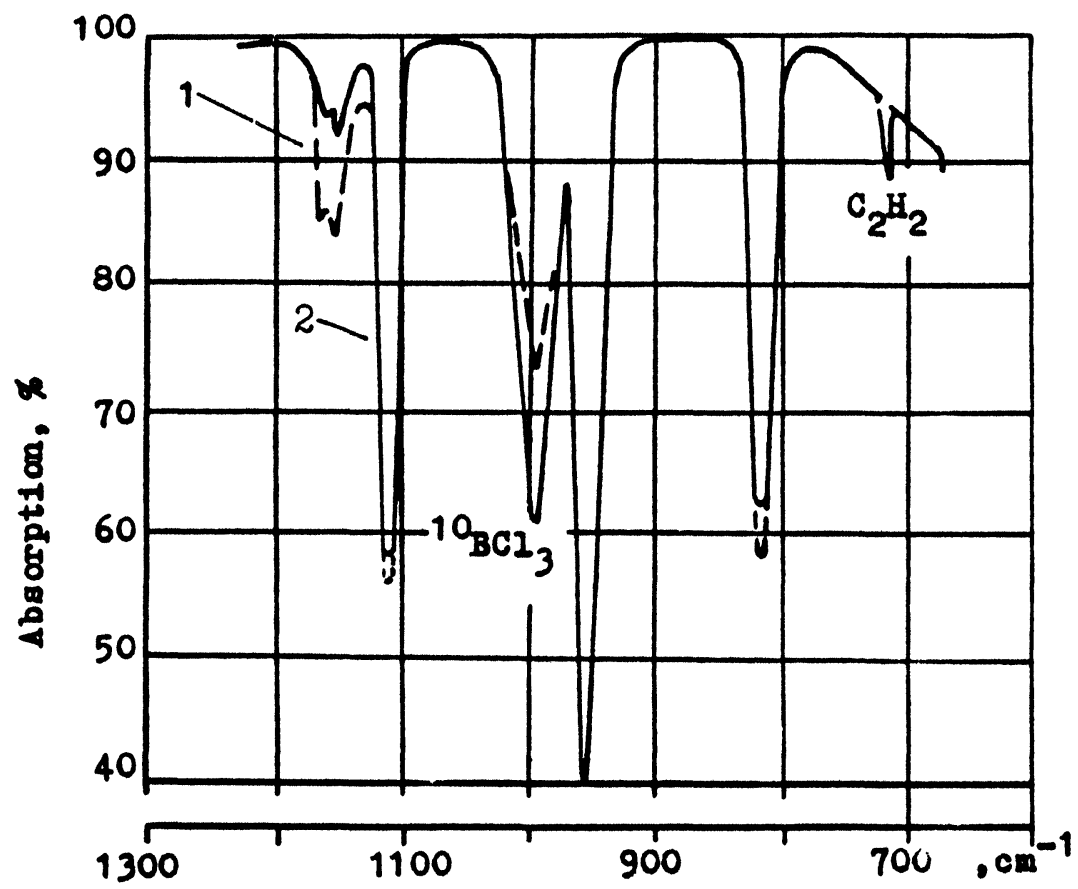

Figure 2 IR linear absorption spectrum. (1) Before the substance irradiation. (2) After the substance irradiation.

portions of the material through the reaction zone the following phenomenon was observed: the input and the output reactor cell windows were covered with a film-like deposit leading to the windows opacity and cracking. All the inner walls of the reactor were covered with powder. The substance deposited more intensely on the output window than on the input one. Thickness of the output window deposit was 2-3 times that of the input window. Deposited substance distribution reflected laser beam intensity fluctuation.

To determine the film elementary composition mass-spectrograms were recorded using RIBER LAS-2000 surface analyzer and a method of secondary ion massspectroscopy (SIMS). For this purpose the film was taken from the sodium chloride monocrystal and transported on to the silica support ai:d inserted into the instrument measuring chamber. It was irradiated in $10^{-8} \mathrm{~Pa}$ vacuum by argon ions accelerated in the electric field. In addition to mass composition SIMS method provided data on the depth of element occurrence in the film. Spectrogram processing showed presence of mass lines of the following elements: boron-10, boron-11, carbon-12, sodium-23, silicon-28, argon-40. Elementary boron with mass number 10 and 11 was the basic element in the film. All the rest mass lines were due to the impurity levels of up to $10^{-4} \mathrm{wt} \%$. Carbon atoms occur because of the secondary chemical reactions between chlorine and acetylene atoms. ${ }^{12}$ Detected sodium atoms were traces of the sodium chloride substrate. Silicon atoms get into the chamber from the holder material. 
Argon atoms were used as the bombarding particles. Figure 3 shows typical SIMS spectrograms of films, deposited on the input and output reactor windows. They vary greatly isotopic content. This fact was verified by the mass-spectrometric study of film composition in МИ-1201 unit modified for solid phase measurements.

These films were also studies by electron diffraction and electron microsopy methods using УЭВМ-100 K unit, working as an electron diffractometer with ДО-2 attachment, and OPTPON EM-10 high-resolution electron microscope (FGR). Electron diffraction analysis of boron films using $75 \mathrm{kV}$ accelerating voltage shows mainly amorphous phase containing crystallites as well (Figure 4). Electronmicroscopic studies at $100 \mathrm{kV}$ accelerating voltage permits to resolve fine amorphous structure of the studied boron film (Figure 5) and to learn that the film contains statistically distributed structural elements-icosahedral boron atom clusters, shortrange order of the received amorphous phase being similar to that of the $\beta$-rombohedral crystalline boron modification.

\section{DISCUSSION}

To explain the observed phenomena we assume that the following processes are imposed in the reactions zone: (a) MP monomolecular decay of molecules into excited fractions in the field of a laser pulse; (b) secondary nonequilibrium reactions during which production of elementary boron is possible in the reaction zone; (c)

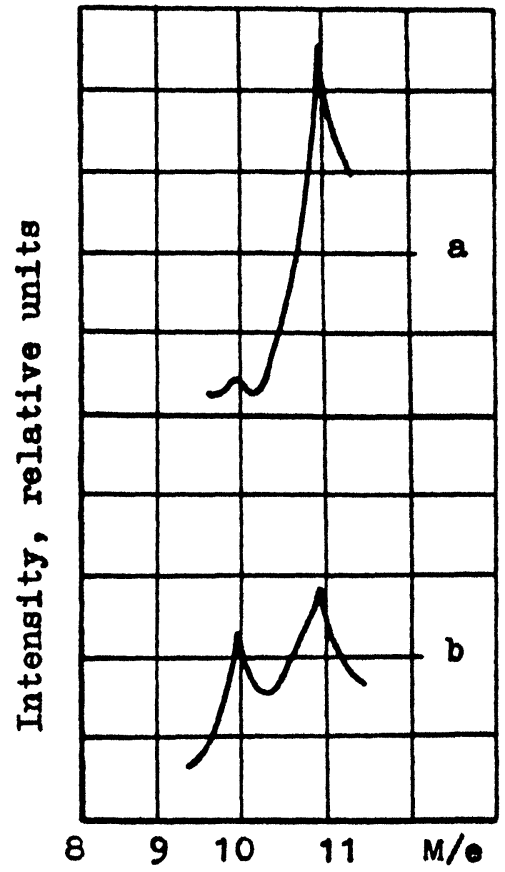

Figure 3 Typical SIMS-spectrogram of the deposited films. (a) On the input window. (b) On the output window. 


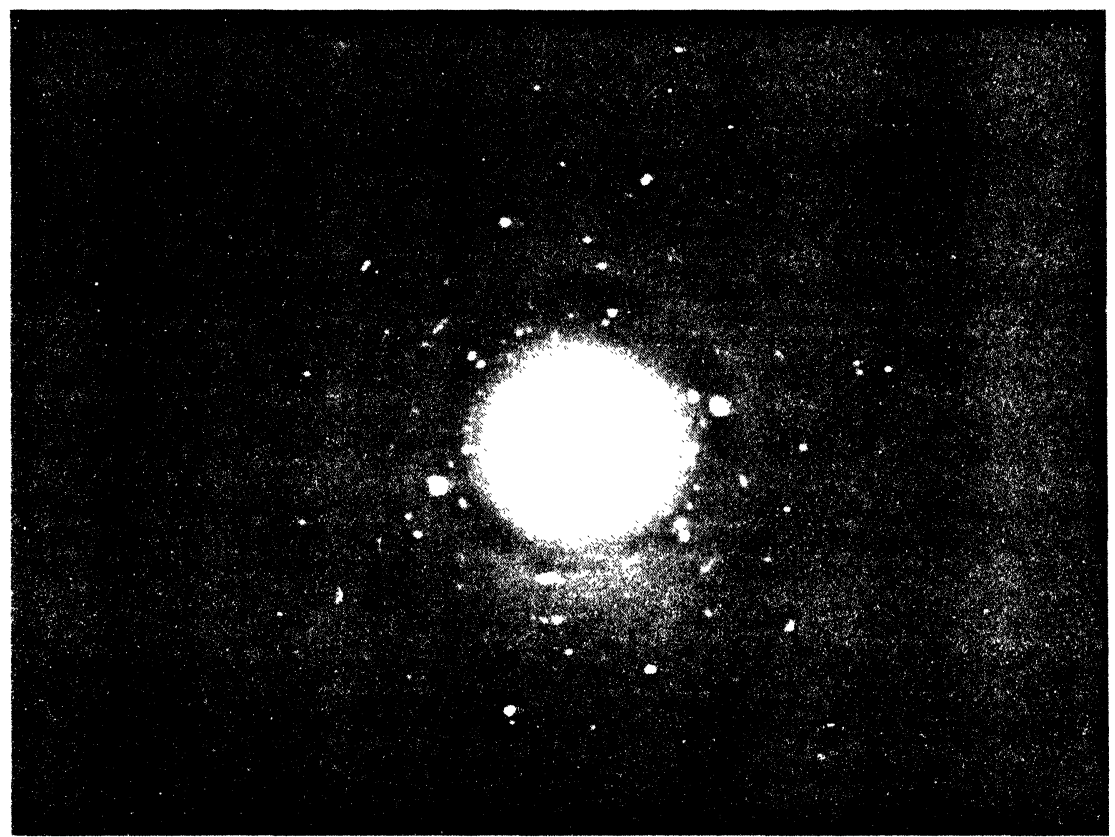

Figure 4 Electrogram of the amorphous boron films with crystalline inclusions.

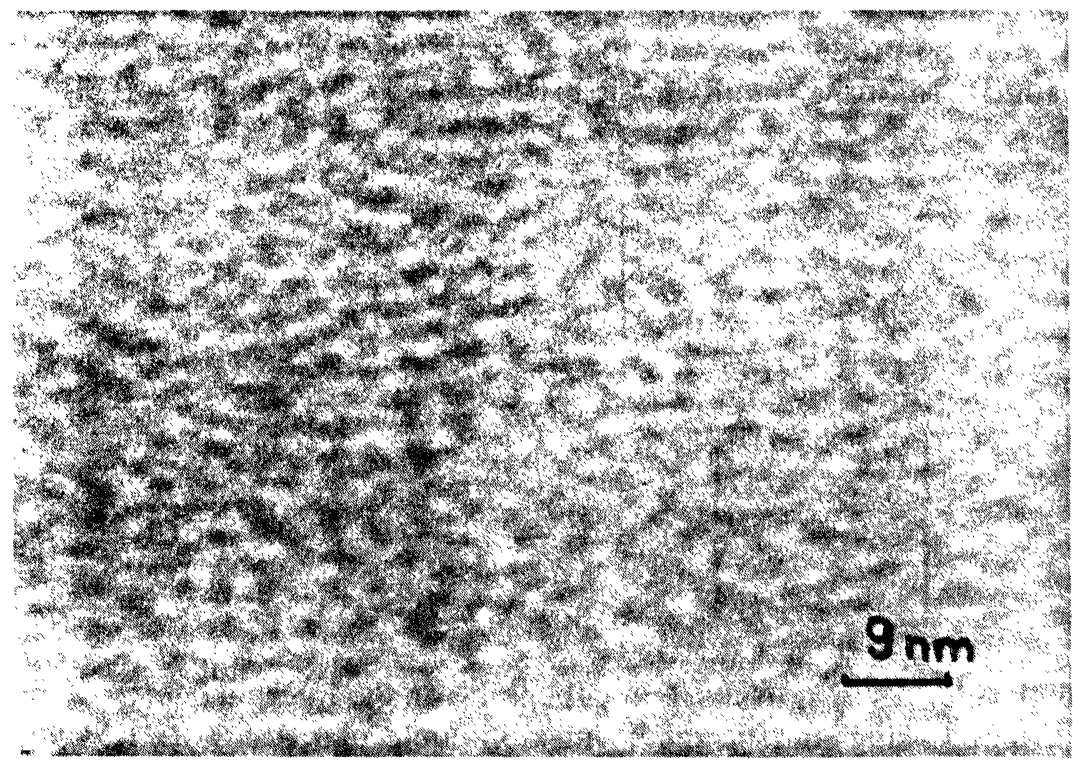

Figure 5 Electron micrograph of the elementary boron amorphous film. 
elementary boron clusters nucleation in the gas-phase; (d) light induced flow along the laser beam path (confirmed by the predominant particle deposition on the output window, compared to that on the input one, and by the boron isotope content difference in these deposits), which overlaps processes (b) and (c) and causes selective mass-transfer.

Let us consider each item separately.

a) Two schemes are possible during monomolecular MP decay simulation:

1) $\mathrm{HClC}=\mathrm{CBCl}_{2} \mathrm{H}$ molecule existing in the form of trans-isomer transforms into cis-form due to IR multiphoton absorption of the pulsed $\mathrm{CO}_{2}$-laser resonance radiation, and then $\mathrm{BCl}_{3}$ splitting off occurs according to monomolecular mechanism

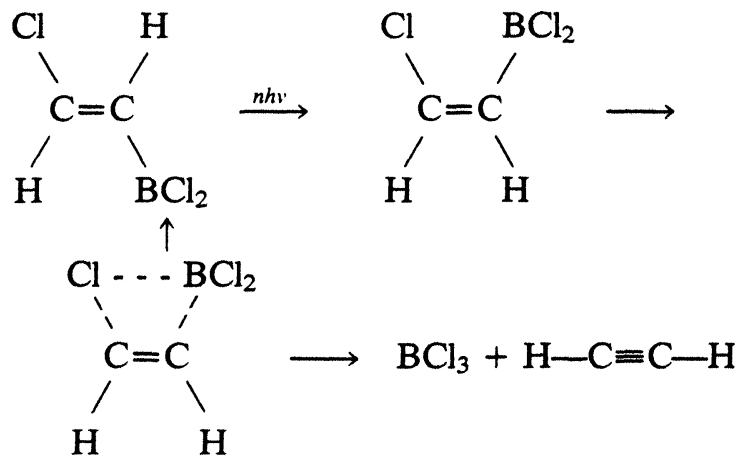

2) As a result of IR multiphoton absorption the possibility arises of the weakest $\mathrm{C}-\mathrm{Cl}$ bond break during molecule transition from normal into active state $\left(E_{\mathrm{C}-\mathrm{Cl}}=\right.$ $392 \mathrm{~J} / \mathrm{mol}$ ), and due to the intramolecular chlorine atom migration intermediate carbene may be produced, which may be rearranged into alkine, producing acetylene and $\mathrm{BCl}_{3}$ during simultaneous break of two $\mathrm{C}-\mathrm{Cl}$ and $\mathrm{C}-\mathrm{BCl}_{2}$ bonds:

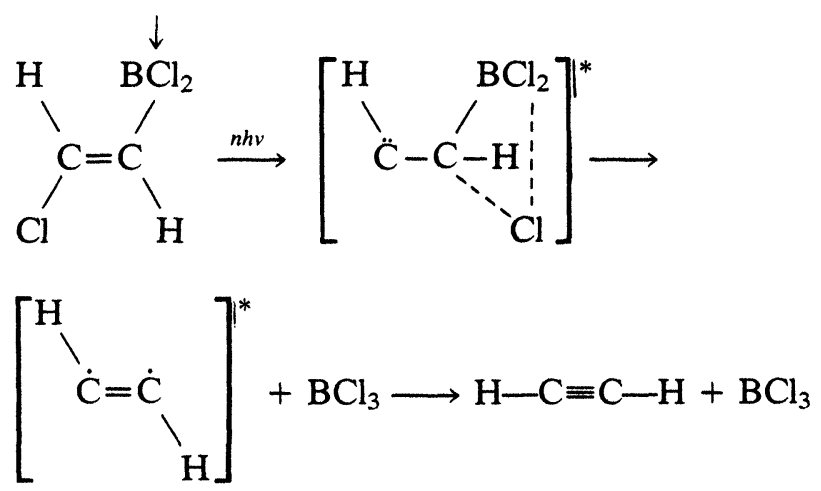

Monomolecular decay rate constant depending on the internal energy of molecules was calculated using statistic theory of monomolecular decay RRKM. ${ }^{13}$ For this purpose we used scheme (2). In our calculations we used the fact, that chlorethylene- 


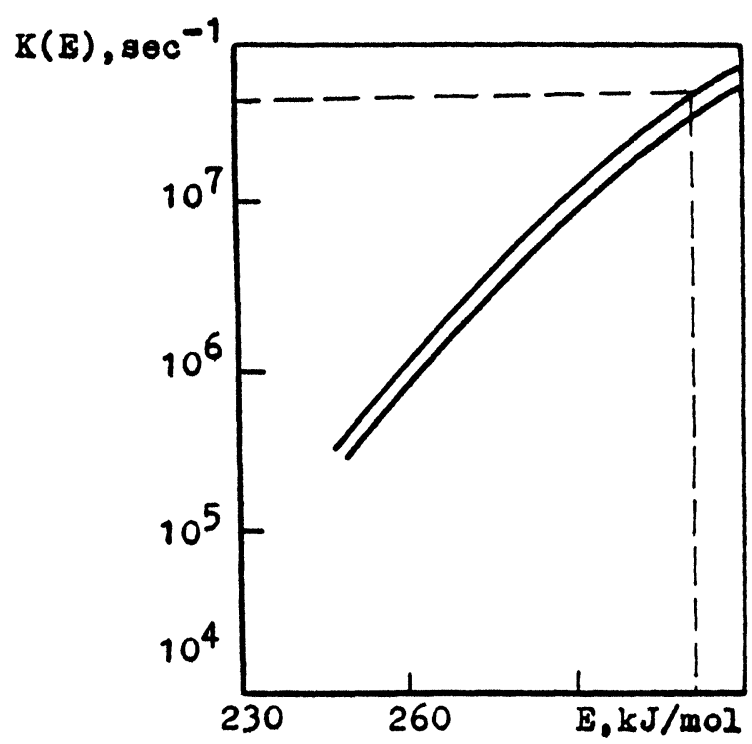

Figure 6 Decay rate constant for $\mathrm{HClC}=\mathrm{CBCl}_{2} \mathrm{H}$ molecule, depending on the internal energy of the molecule.

dichlorborane molecule is characterized by strong asymmetry and has a distant functional additive group $\mathrm{BCl}_{2}$ producing slight perturbation into the basic vibrational frequencies of the molecule. Quantum state densities were calculated in Vitten-Rabinovich approximation. Figure 6 shows the received $k(E)$ dependencies of the both boron isotope modifications. They may be used for estimation of an average energy $E$, at which dissociation of the molecule may occur. The effect of buffer gas on the initial dissociation yield during laser pulse may be used as a quantitative parameter for such estimation. Collisions of the excited molecules with the buffer gas molecules should lead to desactivation, and consequently to the reduction of the dissociation yield probability. When the dissociation yield reduces $e$-fold ( $e$ is a natural logarithm base), then the time interval between collisions of the excited $\mathrm{HClC}=\mathrm{CBCl}_{2} \mathrm{H}$ molecule with the molecules of the buffer gas is similar to the decay time: ${ }^{14}$

$$
\tau_{\mathrm{coll}} \approx \tau_{\mathrm{dec}} \equiv \frac{1}{k(\bar{E})}
$$

The experiments showed that when monoatomic argon buffer gas was added to the initial substance, dissociation yield of ${ }^{19} B$ isotope reduces $e$-fold, if $P_{\mathrm{ar}} \simeq 10^{3} \mathrm{~Pa}$. Taking into account that argon atom diameter $D=3.64 \cdot 10^{-8} \mathrm{~cm},{ }^{15}$ we can receive $\tau_{\text {coll }}=0.2 \cdot 10^{-7} \mathrm{~s}$ for room temperature and pressure $P=10^{3} \mathrm{~Pa}$, and from Eq. (3) $k(\bar{E})=5 \cdot 10^{7} \mathrm{~s}^{-1}$. Knowing the decay rate constant we get from the calculated $K(\bar{E})$ dependencies, that mean decay energy $\bar{E}$ of $\mathrm{HClC}=\mathrm{C}^{10} \mathrm{BCl}_{2} \mathrm{H}$ is $315 \mathrm{~kJ} / \mathrm{mol}$. Overexcitation energy above the dissociation limit $\left(E=E-D_{0}\right)$, remaining 
practically in the dissociation products $(\approx 85 \mathrm{~kJ} / \mathrm{mol})$, is $\approx 7$ IR quantums at laser radiation frequency $v=986.6 \mathrm{~cm}^{-1}$. It is known that molecules get into the vibrational quasicontinuum after absorbing 3-5 IR quantums. ${ }^{1-2}$ It means that decay fractions are already in the vibrational quasicontinuum. Dissociation product $\mathrm{BCl}_{3}$ may resonantly absorb energy in the vibrational quasicontinuum up to the decay into simpler fractions $\mathrm{BCl}_{2}$ and $\mathrm{Cl}$. Further decay down to elementary boron should proceed through the nonradiative way, as proper absorption frequencies of $\mathrm{BCl}_{2}, 700$ $\mathrm{cm}^{-1}, 250 \mathrm{~cm}^{-1}$ and $725 \mathrm{~cm}^{-1}$. are off resonance with IR radiation of $\mathrm{CO}_{2}$-laser.

b) To our mind possible nonequilibrium decay channels of organic boron moleclues down to elementary boron in laser radiation field are connected with bimolecular reations. From the analysis of the available negative results of laser induced bimolecular organic reactions it was concluded in paper, ${ }^{3}$ that both vibrational excitation of each reagent and excitation of translational degrees of freedom are necessary for this purpose. Our case meets these requirements. During the laser pulse $\left(\sim 10^{-7} \mathrm{~s}\right)$ not only excited chlorethylenedichlorborane, but also excited decay fractions $\left(\sim 10^{-8} \mathrm{~s}\right)$ appear in the active zone of the beam. (All of them are excited by the same laser pulse). Besides, translational energy received by the fractions of the monomolecular decay may be sufficient to overcome the activation barrier. ${ }^{16}$ Reactions between the excited fractions and the excited molecules of the "hot" ensemble are the possible channels of elementary boron production. Among the various reaction channels between $\mathrm{BCl}_{3}{ }^{*}, \mathrm{BCl}_{2}{ }^{*}, \mathrm{C}_{2} \mathrm{H}_{2}{ }^{*}, \mathrm{HClC}=\mathrm{CHBCl}_{2}{ }^{*}$, the following reaction is considered the basic one for elementary boron production:

$$
\mathrm{BCl}_{2}{ }^{*}+\mathrm{C}_{2} \mathrm{H}_{2}{ }^{*} \longrightarrow \mathrm{HClC}=\mathrm{CHCl}+\mathrm{B}
$$

Reaction (4) is considered as one of the possible channels, because absorption peaks of dichlorethylene $\mathrm{C}_{2} \mathrm{H}_{2} \mathrm{Cl}_{2}\left(718,895,1175,1270,1225 \mathrm{~cm}^{-1}\right)$ were recorded during the experiments while processing large quantities of the matter. This process is quite probable because it is known, that bonding energy $E_{\mathrm{BCl}-\mathrm{Cl}}<E_{\mathrm{C}-\mathrm{Cl}}\left(E_{\mathrm{BCl}-\mathrm{Cl}}=318\right.$ $\mathrm{J} / \mathrm{mol})$. As bonding energy $E_{\mathrm{C}-\mathrm{H}}>E_{\mathrm{C}-\mathrm{Cl}}\left(E_{\mathrm{C}-\mathrm{H}}=443 \mathrm{~kJ} / \mathrm{mol}\right)$, substitution reaction is less possible than the reaction of adding halogene to alkenes. ${ }^{17}$

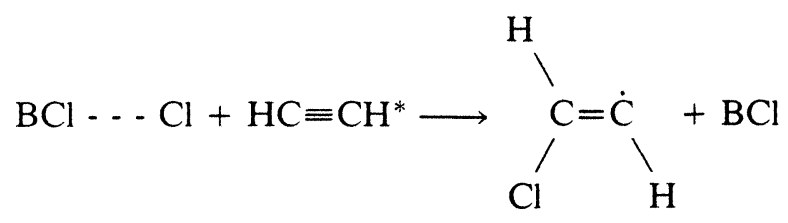

Change of this reaction enthalpy

$$
\Delta \mathrm{H}=-\left(\Delta \mathrm{H}_{\mathrm{Cl} . \ldots \mathrm{CM}=\mathrm{CH}}^{\circ}-\Delta \mathrm{H}_{\mathrm{BCl}-\mathrm{Cl}}^{\circ}\right)=-74 \mathrm{~kJ} / \mathrm{mol} \text {. }
$$

It may be also said about items (a) and (b) that chemical thermometer method is known to be a simple but rather effective method for distinguishing between a nonequilibrium monomolecular laser process and a thermal reaction, according to 
which a dope is added into the basic mixture, the molecules of which do not absorb laser radiation but may undergo monomolecular thermal reaction. Then on the basis of final products ratio we can determine the relation between the nonequilibrium and thermal channels. The molecules of nonresonant isotope component play the part of such molecules in our isotope mixture. They meet all the requirements for the chemical thermometer (except slight nonresonant absorption). Isotope selectivity of the radiative decay preserves, as secondary chemical reactions of elementary boron production proceed mainly between the excited particles. Isotope selectivity of reaction products, determined during the elemental analysis of films, deposited on the optical components, confirms the non-equilibrium character of the studied processes and is a measure of its nonthermality.

c) Boron atoms may coalesce into nuclei during collisions. Parameters directly affecting nucleation kinetics and particle growth in the gas-phase are as follows: reaction zone temperature, heating rate, reactant gas partial pressure etc. ${ }^{16}$ When nucleus production terminates in icosahedron production, a possibility arises of producing $\alpha$ - and $\beta$-modifications of boron. To provide $\beta$-boron modification further coalescence of grains, containing up to seven icosahedra, is necessary. These icosahedra may stick together by surface forces which reduce total surface energy providing contact interfaces.

It is difficult to describe grain nucleation mechanism because of the lack of temperature measurements in the reaction zone (vibrational temperature $T_{v}$ and translational temperature $T$ ). However elementary boron structure should be considered during simulation. Presence of the deposited film structure corresponding to the high-temperature $\beta$-rhombohedral boron structure indicates nucleation of particles with short-range order of positioning atom groups in the reaction zone. This is possibly due to the involvement of particles of "hot" ensemble with high internal energy into the reactions. Grains of elementary boron produced in the reaction zone precipitate as a powder on the inner cell surface and are deposited on the optical windows due to the light induced mass-transfer. When amorphous boron film appears on the optical window, its absorptivity rises and local crystallization of amorphous phase takes place due to the heat release enhancement and film temperature rise. Original amorphous boron structure transforms into $\beta$-rhombohedral crystalline structure having crystallographic parameters characteristic for $\beta$-boron. Laser induced phase transition has similar dynamics with that of the particle growth of the amorphous elementary boron, studied in work. ${ }^{18}$ At first temperature rise provides grouping of statistically distributed boron icosahedra attachment followed by 3-dimensional growth with crystalline structure forming. Figure 7 shows electrone micrograph of coagulation dynamics of $r$ rnn structural elements and crystalline sites formation in the film.

Further substrate temperature rise stimulates $\beta$-boron crystal size growth, forming crystallographic cuttings and joining along the cleavage planes (Figure 8).

Speaking of items (a), (b) and (c) on the whole it should be mentioned, that literature provides papers on semiconductor powder synthesis in laser induced gas-phase reactions. ${ }^{16,25}$ However in contrast to our study they consider mechanisms of laser pyrolysis. 


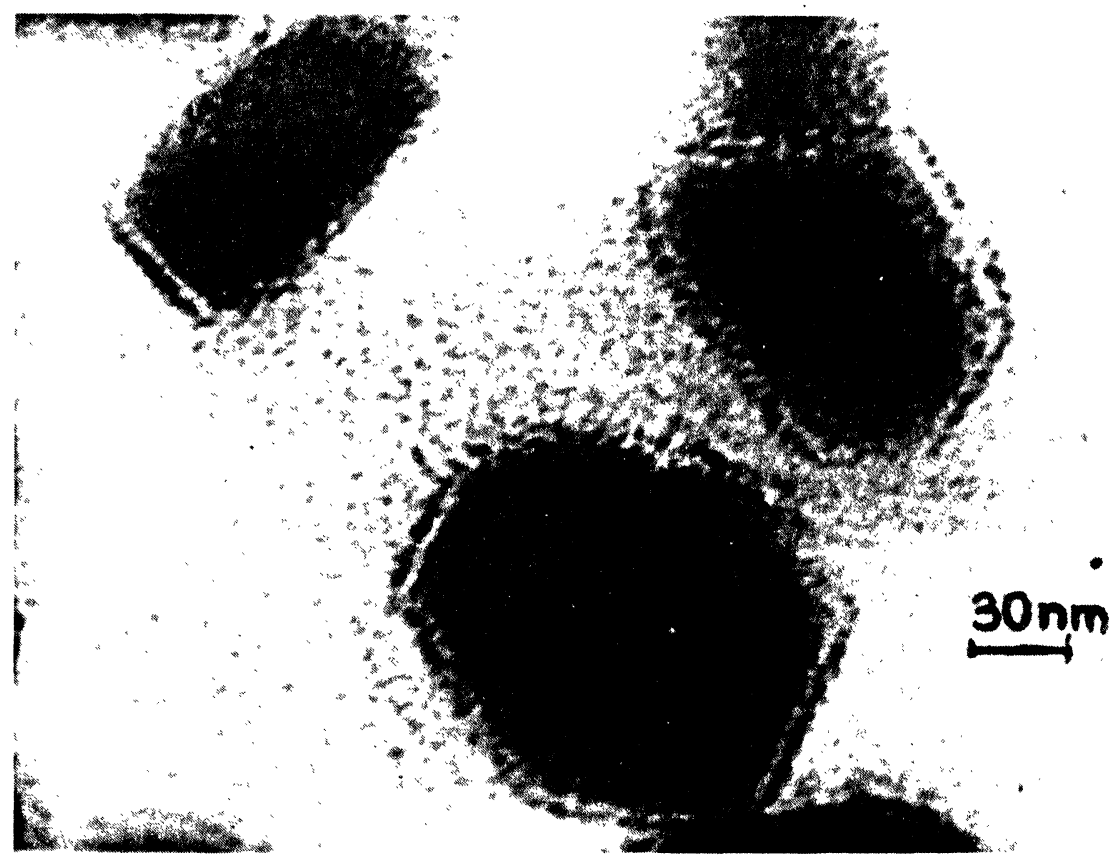

Figure 7 Electron micrograph of the amorphous boron film with crystalline inclusions.

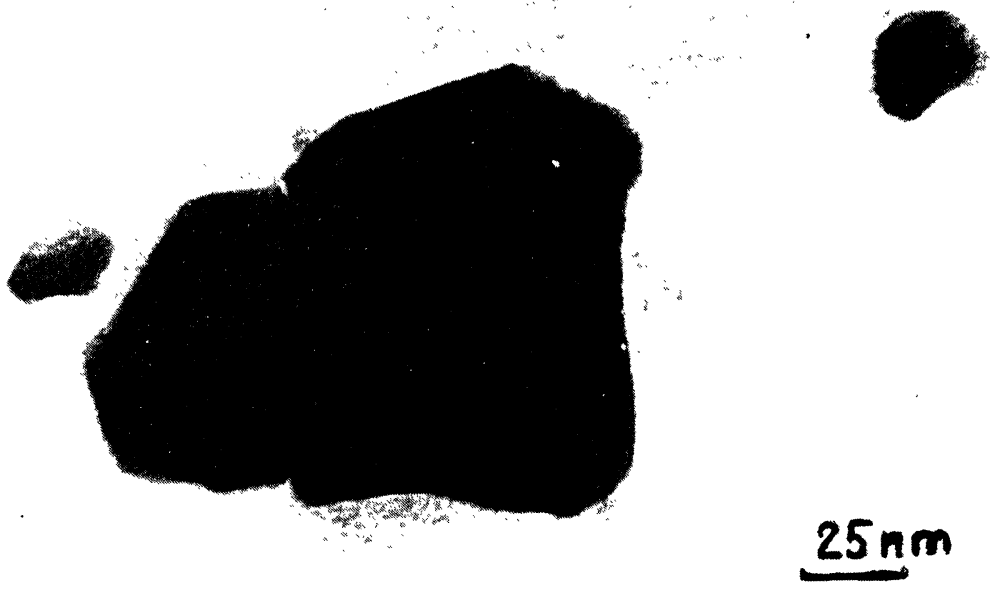

Figure 8 Crystallite, formed by growing together three crystallites along cleavage planes. 
d) As concerns mass-transfer phenomena laser induced thermodiffusion ${ }^{19}$ and light-induced drift (LID) ${ }^{20-22}$ seem to be the most probable effects providing flow motion during IR laser irradiation of molecular gas. Silicon powder deposition on the reactor windows along the laser beam path during $\mathrm{SiH}_{4}$ laser pyrolysis is also discussed in paper. ${ }^{16}$ This fact was attributed to the gas mass-transfer, induced by the local heating by laser and by exothermal chemical reactions.

Special study is necessary to determine which of the above mechanisms provide flow in our case, and it is the object of our further study. However as there are no data on $k_{T}$ diffusion coefficients for $\mathrm{HClC}=\mathrm{CBCl}_{2} \mathrm{H}$ molecules, we cannot compare the sign of the experimentally observed effect with that of the predicted one. But presence of optically thin gas layer (laser energy absorption by molecules of the gas at $1 \mathrm{~m}$ length is only $8-10 \%$ ), and the fact that particle deposition is observed practically in monopulse regime (due to selection of pumping rate and of relative pulse duration) testify against the common thermodiffusion mechanism $\sim \Delta T$. As to the laser thermodiffusion and LID, both of them exploit the effect of particle interaction potential change during excitation. In our case the following could be said about this: $\mathrm{HClC}=\mathrm{CBCl}_{2} \mathrm{H}$ molecule belongs to the class of hydrocarbonunsaturated alkenes. During UV excitation of the most typical representative of this class of molecules the less durable $\pi$-bond is known to break and decompensated electron cloud appears at the unitary $\dot{\mathrm{C}}-\dot{\mathrm{C}}$ bond, which provides repulsion of methylcarbene groups and rotating them out of the initial plane state up to reciprocally-perpendicular planes in space. This changes electron configuration and the received system is characterized by the molecular orbitals of $\mathrm{O}_{2}$ molecule. This change of space form may therefore give rise to the substantial change of interaction potential and consequently of particle transport behaviour. Wave functions of highly excited vibrational levels of the ground electron state and lower vibrational levels of the excited electron state in ethylene overlap during UV excitation, ${ }^{23}$ which gives rise to the assumption that IR MP ethylene excitation provides similar result. The discussed stereoform is assumed to be typical for the excited states of all the alkenes, ${ }^{23}$ and thus for $\mathrm{HClC}=\mathrm{CBCl}_{2} \mathrm{H}$ molecules.

Presence of quasiresonant buffer gas is a positive factor for LID process, ${ }^{22}$ and here this gas consists of nonresonant component molecules containing other boron isotope modifications. But the problem of "hole burning" in the function of rate distribution, necessary for LID effect, is not quite clear in our case.

\section{CONCLUSION}

Experimental study of selective laser-chemistry gas-phase process is discussed, in which elementary boron is the final decay product during initial molecule fission in the field of IR-laser. Final product precipitates as a powder in the reactor and deposits as a film on the optical components in the beam path.

Theoretical model is suggested of such multistage fission due to multiphoton monomolecular decay and subsequent bimolecular reactions between the excited 
decay fragments. Light induced mass transfer is detected along the beam path, and possible mechanisms of this phenomenon are discussed.

It should be mentioned that additional measurements (intermediate radical detection, reaction zone temperature measurements. etc.) must be performed for more detailed process simulation, which is the object of our further study.

\section{References}

1. V. S. Letokhov, Nonlinear Laser Chemistry. Multiple-Photon Excitation (Springer. Berlin. 1983).

2. V. N. Bagratashvili, V. S. Letokhov, A. A. Makarov and E. A. Ryabov, Multiple Photon Infrared Laser Photophysics and Photochemistry. (Gordon and Breach/Harwood. Chur. London. 1985).

3. Laser-induced chemical processes, ed. by J. I. Steinfeld (Plenum Press. New York, 1981).

4. V. N. Bagratashvili. Izv. AN SSSR, 47. 1991 (1983).

5. R. J. Jensen. J. K. Hayes. C. L. Cluff and J. M. Thorne. IEE Journal of Quantum Electronics, 16, 1352 (1980).

6. T. G. Abzianidze. G. I. Abdushelishvili, A. B. Bakhtadze. I. G. Gverdtsiteli. A. V. Kaminski. G. I. Tkeshelashvili and T. B. Tsinadze. Zh. Tekn. Fiz. Pis'ma Red. 8. 1234 (1982).

7. G. I. Abdushelishvili. T. G. Abzianidze, A. S. Egiazarov, G. I. Tkeshelashvili and T. B. Tsinadze, Atomnaia Energia, 57, 203 (1984).

8. G. I. Abdushelishvili, T. G. Abzianidze. A. S. Egiazarov, G. I. Tkeshelashvili and T. B. Tsinadze. Kvantovaia Elektronika, 13. 443 (1986).

9. G. I. Abdushelishvili, A. G. Bakhtadze. P. D. Kervalishvili, G. I. Tkeshelashvili and T. B. Tsinadze. Fiz. Khim. Obr. Mat. 3. $77^{\prime}(1987)$.

10. P. D. Kervalishvili. E. R. Kutelia and G. I. Tkeshelashvili. Fiz. Khim. Obr. Mat. 3. 73 (1987).

11. M. M. Emmanuel and D. G. Knorre, Course of Chemical Kinetics (Vysshaya Shkola. Moscow, 1984. in Russian),

12. S. S. Zaitsev, General Chemistry (Vysshaia Shkola, Moscow, 1983. in Russian).

13. P. J. Robinson and K. A. Holbrook, Unimolecular Reactions (Wiley Interscience, London. New York, Sydney. Toronto, 1972).

14. V. V. Vyzhin and A. K. Petrov, Ju. N. Molin. Dokl. AN SSSR, 243, 129 (1978).

15. E. W. McDaniel, Collision Phenomena in Ionized Gases (John Wiley and Sons. New York, London. Sydney, 1964):

16. J. S. Haggerty and W. R. Cannon, see in Ref. 3.

17. A. L. Ternay, Jr., Contemporary Organic Chemistry (W. B. Saunders Company. Philadelphia, London. Toronto, 1979).

18. P. D. Kervalishvili, E. R. Kutelia and T. A. Dzigrashvili, Fiz. Tverd. Tela, 27, 1414 (1985).

19. N. V. Karlov. Ju. N. Petrov, A. M. Prokhorov and I. V. Fyodorov, Zh. Tekn. Fiz. 48. 2333 (1978).

20. F. Kh. Gelmukhanov and A. M. Shalagin, Zh. Ekap. Teor. Fiz. 78, 1672 (1980).

21. A. M. Dykhne and A. N. Starostin. Zh. Eksp. Teor. Foz. 79. 1211 (1980).

22. F. Kh. Gelmukhanov and C. G. Telegin, Zh. Eksp. Teor. Fiz. 80, 974 (1981).

23. J. A. Barltrop and J. D. Coyle. Excited States in Organic Chemistry (John Wiley and Sons. London, New York. Sydney, Toronto, 1975).

24. A. J. Merer and R. S. Mulliken, Chem. Rev. 69, 369 (1969). 